gr-qc/0505039

BRX TH-563

\title{
Curvature invariants of static spherically symmetric geometries
}

\author{
S. Deser ${ }^{1}$ and A.V. Ryzhov ${ }^{2}$ \\ Department of Physics, Brandeis University \\ Waltham, MA 02454, USA
}

\begin{abstract}
We construct all independent local scalar monomials in the Riemann tensor at arbitrary dimension, for the special regime of static, spherically symmetric geometries. Compared to general spaces, their number is significantly reduced: the extreme example is the collapse of all invariants $\sim \mathrm{Weyl}^{k}$, to a single term at each $k$. The latter is equivalent to the Lovelock invariant $\mathcal{L}_{k}$. Depopulation is less extreme for invariants involving rising numbers of Ricci tensors, and also depends on the dimension. The corresponding local gravitational actions and their solution spaces are discussed.
\end{abstract}

\footnotetext{
${ }^{1}$ Email: deser@brandeis.edu

${ }^{2}$ E-mail: ryzhovav@brandeis.edu
} 


\section{Introduction}

Our subject, construction and enumeration of local curvature invariants for special geometries, i.e. scalars of the form $\left(R_{\mu \nu \alpha \beta}\right)^{k}$ in arbitrary dimension $D$, has both mathematical and physical interest. Two physical applications of the general scheme stand out: the first is to understand geometrical corrections to general relativity, which is "merely" the lowest order term of an effective series of loop or string corrections. For this purpose, an accessible grid of candidate terms is desirable, and indeed an enormous literature has accumulated just on the three quadratic possibilities, $R^{2}, R_{\mu \nu} R^{\mu \nu}, R_{\mu \nu \alpha \beta} R^{\mu \nu \alpha \beta}$. A second, related, application is to list allowed local counterterms in specific quantum models, such as supergravity. The first example, that of $D=4, \mathcal{N}=1$ SUGRA, produced an allowed supersymmetric counterterm, the square of the quadratic Bel-Robinson tensor $T_{\mu \nu \alpha \beta} \sim(R R)_{\mu \nu \alpha \beta}$ at 3-loop order [1]. More recently, it became possible to actually compute explicitly the 2-loop divergences in $D=11$ SUGRA [2], the bosonic part of which was also quartic in curvature (and in four-form field strength). Here again, knowledge of the basis for quartics was a useful tool [3,4]. Meanwhile, on the mathematical side, an algorithmic way to find all independent monomial scalars in Riemann ${ }^{k}$, along with a number of illuminating explicit examples, ${ }^{1}$ was given in [5]. That work illustrated the discouragingly rapid rise of their number with $k$, and to a lesser extent, $D$.

Once the basis of possible corrections is established, there are a number of immediate physical questions one may ask. The most obvious is that of the solutions to the effective actions: will they provide surprises about horizons, naked singularities and other postSchwarzschildean behavior? Here, of course, the only hope of obtaining solutions lies in the simplest possible geometries, namely the static spherically symmetric ones; this question is one of our major motivations. So, apart from a brief excursion away from static spherically symmetric geometries in Section 6 to show how intractable things become for less symmetric spaces, all work below is restricted to static spherically symmetric geometries.

We will establish a decomposition of the curvature tensor into the Weyl and Ricci parts to provide a simple analysis of all generic local curvature invariants. As a result, we will note a dramatic decrease in the number of independent terms and an increase in their transparency. In particular, the general classification will be governed more by the relative powers of Weyl and Ricci components, rather than by the overall monomial power. We will then be able to discuss classes, rather than individual terms, thereby

\footnotetext{
${ }^{1}$ Unlike [5], we will not consider invariants involving explicit derivatives, such as $R^{n} \nabla^{2 p} R^{m}$, although they will certainly appear in any physical context alongside their algebraic counterparts $R^{n+m+p}$.
} 
helping navigate the sea of effective actions and their solutions.

\section{Symmetries and definitions}

We first introduce notation that will make the basis elements $\left(R_{\mu \nu \alpha \beta}\right)^{k}$ as easily computable as possible in arbitrary dimensions ${ }^{2} D=n+2 \geq 4$. Our metric is expressed in Schwarzschild gauge:

$$
d s^{2}=-e^{2 \Phi(r)} d t^{2}+e^{2 \Lambda(r)} d r^{2}+r^{2} d \Omega_{n}^{2}=-\omega_{\hat{t}}^{2}+\omega_{\hat{r}}^{2}+\sum_{i=1}^{n} \omega_{\hat{\imath}} \omega_{\hat{i}}
$$

where the orthonormal basis one-forms are

$$
\omega_{\hat{t}}=-e^{\Phi} d t, \quad \omega_{\hat{r}}=e^{\Lambda} d r
$$

The curvature 2 -form is

$$
\mathcal{R}^{\mu}{ }_{\nu} \equiv d \omega^{\mu}{ }_{\nu}+\omega^{\mu}{ }_{\alpha} \wedge \omega^{\alpha}{ }_{\nu}=\frac{1}{2} R^{\mu}{ }_{\nu \alpha \beta} \omega^{\alpha} \wedge \omega^{\beta}, \quad \omega^{\mu}{ }_{\nu}=\Gamma^{\mu}{ }_{\nu \alpha} \omega^{\alpha}, \quad \omega_{\mu \nu}=g_{\mu \alpha} \omega^{\alpha}{ }_{\nu} .
$$

Using the identities

$$
0=d \omega^{\mu}+\omega_{\nu}^{\mu} \omega^{\nu}, \quad d g_{\mu \nu}=\omega_{\mu \nu}+\omega_{\nu \mu}
$$

and the orthonormal basis (2.2), it is easy to find the connection one-forms $\omega_{\hat{\mu} \hat{\nu}}=-\omega_{\hat{\nu} \hat{\mu}}$ :

$$
\omega_{\hat{t} \hat{r}}=-\Phi^{\prime} e^{-\Lambda} \omega^{\hat{t}}, \quad \omega_{\hat{i} \hat{r}}=\frac{1}{r} e^{-\Lambda} \omega^{\hat{i}}, \quad \omega_{\hat{t} \hat{i}}=0 .
$$

The resulting $\mathcal{R}^{\mu}{ }_{\nu}$ for $D=4$ are given in [6]:

$$
\begin{aligned}
& \mathcal{R}_{\hat{r}}^{\hat{t}}=e^{-2 \Lambda}\left[\Phi^{\prime \prime}+\left(\Phi^{\prime}\right)^{2}-\Phi^{\prime} \Lambda^{\prime}\right] \omega^{\hat{r}} \wedge \omega^{\hat{t}} \equiv A(r) \omega^{\hat{r}} \wedge \omega^{\hat{t}}, \\
& \mathcal{R}_{\hat{i}}^{\hat{t}}=e^{-2 \Lambda}\left[-\frac{1}{r} \Phi^{\prime}\right] \omega^{\hat{t}} \wedge \omega^{\hat{i}} \equiv B(r) \omega^{\hat{t}} \wedge \omega^{\hat{i}}, \\
& \mathcal{R}_{\hat{i}}^{\hat{r}}=e^{-2 \Lambda}\left[\frac{1}{r} \Lambda^{\prime}\right] \omega^{\hat{r}} \wedge \omega^{\hat{i}} \equiv C(r) \omega^{\hat{r}} \wedge \omega^{\hat{i}}, \\
& \mathcal{R}_{\hat{j}}^{\hat{i}}=\frac{1}{r^{2}}\left[1-e^{-2 \Lambda}\right] \omega^{\hat{i}} \wedge \omega^{\hat{j}} \equiv \psi(r) \omega^{\hat{i}} \wedge \omega^{\hat{j}} ;
\end{aligned}
$$

they are in fact dimension-independent. The functions $A, B, C$, and $\psi$, defined in (2.6), are the only combinations of $\Lambda(r)$ and $\Phi(r)$ appearing in the Riemann tensor.

\footnotetext{
${ }^{2}$ In $D=3$, the Weyl tensor vanishes identically, Riemann and Ricci being equivalent (double duals) to each other; in $D=2$, only the scalar curvature $R_{\mu}{ }^{\mu}$ remains.
} 
The $\{A, B, C, \psi\}$ are algebraically, but not functionally, independent; for example, $C(r)=\frac{1}{2 r}\left(r^{2} \psi(r)\right)^{\prime}$. When $A$ and $B$ are used for constructing actions, we can integrate them by parts:

$$
\begin{aligned}
\int_{0}^{\infty} d r r^{n} e^{\Phi+\Lambda} B \Psi & =\int_{0}^{\infty} d r r^{n} e^{\Phi+\Lambda}\left\{\left(\frac{1}{r^{2}}-\psi\right)\left[(n-1) \Psi+r \Psi^{\prime}\right]-C \Psi\right\}, \\
\int_{0}^{\infty} d r r^{n} e^{\Phi+\Lambda} A \Psi & =\int_{0}^{\infty} d r r^{n} e^{\Phi+\Lambda}\left\{B\left[n \Psi+r \Psi^{\prime}\right]\right\}
\end{aligned}
$$

where $\Psi=\Psi(r)$ is an arbitrary function. The following operator relations allow us to express any monomial involving either a single $A$ or a single $B$ (but not both, and no higher powers of these) and an arbitrary number of $C$ 's and $D$ 's in terms of the single function $\psi$ and its derivatives:

$$
C=\psi+\frac{1}{2} r \psi^{\prime}, B \sim\left(\frac{1}{r^{2}}-\psi\right)\left[(n-1)+r \frac{d}{d r}\right]-C, A \sim B\left[n+r \frac{d}{d r}\right]
$$

where " " means "up to integrating by parts with measure $d r r^{n} e^{\Phi+\Lambda} . "$ The Einstein action is immediately obtained using (2.8),

$$
S_{1}=\int \sqrt{g}(R+\lambda)=\int d t \int d^{n} \Omega \int_{0}^{\infty} d r e^{\Phi+\Lambda}\left[\left(n \psi(r)+\frac{1}{n+1} \lambda\right) r^{n+1}\right]^{\prime}
$$

whose extrema ${ }^{3}$ are obviously the Schwarzschild-deSitter metrics

$$
\Phi=-\Lambda=\frac{1}{2} \ln \left[1-\frac{\zeta}{r^{n-1}}+\frac{\lambda r^{2}}{n(n+1)}\right] .
$$

\section{$3 \quad$ Projectors and components}

The metric (2.1) separates the space-time indices $\mu$ into $t, r$, and the angular $i=$ $1, \ldots, n$. The tensors

$$
\begin{aligned}
\tau_{\hat{\mu}}^{\hat{\nu}} & \equiv \delta_{\hat{\hat{t}}}^{\hat{t}} \delta_{\hat{\nu}}^{\hat{\nu}}=\operatorname{diag}(1,0,0, \ldots, 0), \\
\rho_{\hat{\mu}}^{\hat{\nu}} & \equiv \delta_{\hat{\mu}}^{\hat{r}} \delta_{\hat{r}}^{\hat{\nu}}=\operatorname{diag}(0,1,0, \ldots, 0), \\
\sigma_{\hat{\mu}}^{\hat{\nu}} & \equiv \sum_{i=1}^{n} \delta_{\hat{\mu}}^{\hat{i}} \delta_{\hat{i}}^{\hat{\nu}}=\operatorname{diag}(0,0,1, \ldots, 1)
\end{aligned}
$$

conveniently summarize this decomposition. They are orthogonal projectors,

$$
\tau \tau=\tau, \quad \rho \rho=\rho, \quad \sigma \sigma=\sigma ; \quad \tau \rho=\tau \sigma=\rho \sigma=0,
$$

\footnotetext{
${ }^{3}$ This reduction, justified in [7], has also proved quite useful for quadratic curvature models [8].
} 
and multiply as matrices, $(\tau \tau)_{\hat{\mu}}^{\hat{\hat{\alpha}}}=\tau_{\hat{\mu}}^{\hat{\nu}} \tau_{\hat{\nu}}^{\hat{\alpha}}=\tau_{\hat{\mu}}^{\hat{\hat{}}}$ etc. Their traces are

$$
\operatorname{tr} \tau=1, \quad \operatorname{tr} \rho=1, \quad \operatorname{tr} \sigma=n .
$$

In an orthonormal frame, indices are raised and lowered by the Minkowski metric, e.g. $\tau_{\hat{\mu} \hat{\nu}}=\tau_{\hat{\mu}}^{\hat{\alpha}} \eta_{\hat{\alpha} \hat{\nu}}$. Since the (3.1) are symmetric matrices, we need not worry about index order, and can write $\tau_{\hat{\mu}}^{\hat{\nu}}$ for $\tau_{\hat{\mu}}^{\hat{\nu}}$, etc.

The projectors (3.1) also provide a compact notation for the curvatures. The Riemann tensor is

$$
R_{\hat{\alpha} \hat{\mu} \hat{\beta}}^{\hat{\mu}}=2\left[-A \cdot 2 \tau_{[\hat{\alpha}}^{[\hat{\mu}} \rho_{\hat{\beta}]}^{\hat{\nu}]}+B \cdot 2 \tau_{[\hat{\alpha}}^{[\hat{\mu}} \sigma_{\hat{\beta}]}^{\hat{\nu}]}+C \cdot 2 \rho_{[\hat{\alpha}}^{[\hat{\mu}} \hat{\left.\sigma_{\hat{\beta}]}\right]}+\psi \cdot \sigma_{[\hat{\alpha}}^{[\hat{\mu}} \sigma_{\hat{\beta}]}^{\hat{\nu}]}\right],
$$

while Ricci and Weyl can be written as

$$
\begin{aligned}
R_{\hat{\mu}}^{\hat{\nu}} & =F \tau_{\hat{\mu}}^{\hat{\nu}}+G \rho_{\hat{\mu}}^{\hat{\nu}}+H \sigma_{\hat{\mu}}^{\hat{\nu}} \\
C_{\hat{\alpha} \hat{\beta}}^{\hat{\mu} \hat{\nu}} & =-2 \chi \frac{n-1}{n+1}\left[2 \tau_{[\hat{\alpha}}^{[\hat{\mu}} \rho_{\hat{\beta}]}^{\hat{\nu}]}-2 \frac{1}{n}\left(\tau_{[\hat{\alpha}}^{[\hat{\mu}} \sigma_{\hat{\beta}]}^{\hat{\hat{\beta}}]}+\rho_{[\hat{\alpha}}^{[\hat{\mu}} \sigma_{\hat{\beta}]}^{\hat{\nu}]}\right)+\frac{2}{n(n-1)} \sigma_{[\hat{\alpha}}^{[\hat{\mu}} \sigma_{\hat{\hat{\beta}}]}^{\hat{\nu}]}\right]
\end{aligned}
$$

where

$$
F \equiv n B-A, G \equiv n C-A, H \equiv B+C+(n-1) \psi,
$$

are the Ricci components, and

$$
\chi \equiv A+B+C-\psi
$$

is the only function appearing in the definition of Weyl. Here, the brackets denote (normalized) antisymmetrization, $2 \sigma_{[\hat{\alpha}}^{[\hat{\mu}} \sigma_{\hat{\beta}]}^{\hat{\nu}]}=\sigma_{\hat{\alpha}}^{\hat{\mu}} \sigma_{\hat{\beta}}^{\hat{\nu}}-\sigma_{\hat{\beta}}^{\hat{\mu}} \sigma_{\hat{\alpha}}^{\hat{\nu}}$, etc. The (four-index) structures $2 \tau_{[\hat{\alpha}}^{[\hat{\mu}} \rho_{\hat{\beta}]}^{\hat{\nu}]}$, $2 \tau_{[\hat{\alpha}}^{[\hat{\mu}} \sigma_{\hat{\beta}]}^{\hat{\nu}]}, 2 \rho_{[\hat{\alpha}}^{[\hat{\mu}} \sigma_{\hat{\beta}]}^{\hat{\nu}]}$, and $\sigma_{[\hat{\alpha}}^{[\hat{\mu}} \sigma_{\hat{\beta}]}^{\hat{\nu}]}$ are also orthogonal projectors, e.g., $\sigma_{[\hat{\alpha}}^{[\hat{\mu}} \sigma_{\hat{\beta}]}^{\hat{\nu}]} \sigma_{[\hat{\epsilon}}^{[\hat{\alpha}} \sigma_{\hat{\gamma}]}^{\hat{\beta}]}=\sigma_{[\hat{\epsilon}}^{[\hat{\mu}} \sigma_{\hat{\gamma}]}^{\hat{\nu}]}$. Powers of Riemann, Ricci, and Weyl have similar form and are discussed in the Appendix.

To list all independent invariants, we first organize them according to the number of Weyls (i.e., the power of $\chi$ ), and then eliminate redundancies at every given power of $\chi$. The locations of the indices for Weyl and Ricci (before contractions into scalars) can be fixed once and for all as $C_{\hat{\alpha} \hat{\nu} \hat{\beta}}$ and $R_{\hat{\mu}}^{\hat{\nu}}$; then, one can use (3.5) and (3.6) without having to worry about raising or lowering their indices. All other configurations are linear combinations of these. The resulting invariants may still be linearly dependent; for example, we have not made use of the cyclic symmetry $R_{\mu[\nu \alpha \beta]}=0$. So one may need to eliminate the extras by hand. Invariants made out of the Riemann tensor, or equivalently its Weyl and Ricci components, can be written in terms of products of (3.1), and contracted using (3.2) and (3.3); these are the only tools required for our calculations. As (3.6) is symmetric under $\tau \leftrightarrow \rho$, the resulting invariants will be symmetric under $F \leftrightarrow G$; see (3.5)-(3.6). 


\section{Lovelock invariants}

To connect with earlier studies of specific actions, it is convenient to formulate Weyl ${ }^{k}$ monomials in terms of the Euler-Gauss-Bonnet-Lovelock invariants



with

$$
\delta_{\alpha_{1} \alpha_{2} \ldots \alpha_{r}}^{\mu_{1} \mu_{2} \ldots \mu_{r}} \equiv \frac{1}{r !} \sum_{\gamma \in S_{r}} \operatorname{sign}(\gamma) \delta_{\alpha_{\gamma(1)}}^{\mu_{1}} \delta_{\alpha_{\gamma(2)}}^{\mu_{2}} \ldots \delta_{\alpha_{\gamma(r)}}^{\mu_{r}}
$$

see e.g. [9]. Here, $\delta$ is totally antisymmetric in its upper and lower indices separately, and the normalization is chosen to make $\delta$ a projector: $\delta_{\mu_{1} \mu_{2} \ldots \mu_{r}}^{\nu_{1} \nu_{2} \ldots \nu_{r}} \delta_{\alpha_{1} \alpha_{2} \ldots \alpha_{r}}^{\mu_{1} \mu_{2} \ldots \mu_{r}}=\delta_{\alpha_{1} \alpha_{2} \ldots \alpha_{r}}^{\nu_{1} \nu_{2} \ldots \nu_{r}}$. When $D=2 k, \mathcal{L}_{k}$ is a total divergence, and it vanishes identically for $D<2 k$.

The $\mathcal{L}_{k}$ are easily evaluated. Antisymmetry of the Riemann tensor (3.4) in its upper and lower indices, reduces (4.1) to sums of

$$
\delta_{\beta_{1} \ldots \gamma_{1} \ldots \epsilon_{1} \ldots}^{\mu_{1} \ldots \nu_{1} \ldots \alpha_{1} \ldots}\left(\tau_{\mu_{1}}^{\beta_{1}} \ldots \tau_{\mu_{p}}^{\beta_{p}}\right)\left(\rho_{\nu_{1}}^{\gamma_{1}} \ldots \rho_{\nu_{q}}^{\gamma_{q}}\right)\left(\sigma_{\alpha_{1}}^{\epsilon_{1}} \ldots \sigma_{\alpha_{r}}^{\epsilon_{r}}\right)=\frac{n !}{(p+q+r) !(1-p) !(1-q) !(n-r) !} .
$$

Permutations mixing indices from different groups $(\{\mu\},\{\nu\},\{\alpha\})$ vanish, since the projectors $\tau, \rho$, and $\sigma$ are mutually orthogonal; their traces are given in (3.3). For (4.3) to be nonzero, we must have $2 k=p+q+r \leq n+2=D$. From the explicit form of (3.4) we find that $\mathcal{L}_{k}$ is the specific combination

$$
\mathcal{L}_{k}=\zeta_{k}^{n}\left[(n-2 k+1) \psi^{k}+2 k(B+C) \psi^{k-1}+\frac{2 k}{(n-2 k+2)}(2(k-1) B C-A \psi) \psi^{k-2}\right],
$$

where the prefactor $\zeta_{k}^{n} \equiv \frac{2^{k} n !}{(2 k) !(n-2 k+1) !}\left(\right.$ so $\zeta_{k}^{n}=0$ for $\left.n+2 \leq 2 k\right)$. Using (2.8) to integrate by parts, the $\mathcal{L}_{k}$ give rise to uniformly nice actions

$$
S_{\mathcal{L}}=\int d^{D} x \sqrt{g} \sum_{k} a_{k} \mathcal{L}_{k}=\int d t \int d^{n} \Omega \int_{0}^{\infty} d r e^{\Phi+\Lambda}\left[r^{n+1} \sum_{k} a_{k} \zeta_{k}^{n} \psi(r)^{k}\right]^{\prime}
$$

for any set of coefficients $a_{k}$. The attractive properties of the Einstein action (2.9) are preserved: "on-shell," $\Phi+\Lambda$ is constant and $\psi(r)$ satisfies an algebraic (rather than a differential) equation $[10,11]$. This displays the fact that general $S_{\mathcal{L}}$ actions contain only two derivatives, further reducing to just one in Schwarzschild coordinates.

The four independent components of $R_{\hat{\nu} \hat{\alpha} \hat{\beta}}^{\hat{\beta}}$ can be expressed as linear combinations of 
the three components of $R_{\hat{\mu} \hat{\nu}}$ and the single independent component of the Weyl tensor:

$$
\left(\begin{array}{l}
A \\
B \\
C \\
\psi
\end{array}\right)=\frac{1}{n(n+1)}\left(\begin{array}{c}
-n(F+G)+n H+n(n-1) \chi \\
n F-G+H+(n-1) \chi \\
-F+n G+H+(n-1) \chi \\
-(F+G)+(n+2) H-2 \chi
\end{array}\right)
$$

From (4.6) it immediately follows that an arbitrary Riemann ${ }^{k}$ invariant can be generally written schematically as



In this fashion, powers of $\chi$ multiplying structures involving only components of the Ricci

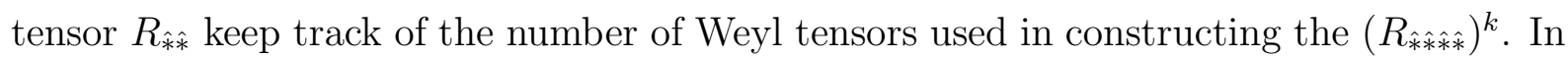
particular, $\mathcal{L}_{k}$ can be described in an alternate way to (4.4),

$$
\mathcal{L}_{k}=a_{k}^{n} \chi^{k}+\chi^{k-1}\left(R_{\hat{*} \hat{*}}\right)+\chi^{k-2}\left(R_{\hat{*} \hat{*}}\right)^{2}+\ldots+\left(R_{\hat{*} \hat{k}}\right)^{k}
$$

with $a_{k}^{n}=\frac{(n+1) !(k-1)(n k+k-n-2)}{(2 k) !(n-2 k+2) !}\left(\frac{-4}{n(n+1)}\right)^{k}$, and, as an immediate corollary of (4.7) and (4.8)

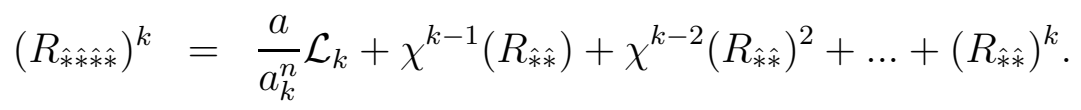

Any Riemann ${ }^{k}$ invariant is proportional to $\mathcal{L}_{k}$ modulo terms involving at least one occurrence of $R_{\hat{*} \hat{*}}$. In general, all powers of $\chi$ will be present, so it is not possible to express arbitrary scalars in terms of only Riccis and the highest Lovelock.

\section{$5 \quad$ Specific examples and general patterns}

Consider now some explicit examples. The most trivial one is $k=0$, the constant (cosmological) term

$$
\mathcal{L}_{0}=1
$$

At $k=1$, the Ricci scalar is the unique invariant

$$
\mathcal{L}_{1}=R
$$

At $k=2$, there are three invariants:

$$
I_{1}^{2} \equiv R^{2}, I_{2}^{2} \equiv R_{\mu \nu} R^{\mu \nu}, I_{3}^{2} \equiv R_{\mu \nu \alpha \beta} R^{\mu \nu \alpha \beta}
$$


whose Lovelock combination (4.1) is

$$
\mathcal{L}_{2}=\frac{1}{6}\left[R_{\mu \nu \alpha \beta} R^{\mu \nu \alpha \beta}-4 R_{\mu \nu} R^{\mu \nu}+R^{2}\right] .
$$

There are still 3 invariants we can construct out of Ricci and Weyl:

$$
\operatorname{tr} R^{2}=F^{2}+G^{2}+n H^{2}, \quad(\operatorname{tr} R)^{2}=(F+G+n H)^{2} ; \quad \operatorname{tr} C^{2}=4 \chi^{2} \frac{n-1}{n+1} .
$$

At order $k=3$, the basis of curvature invariants (for general metrics) has eight members [5]:

$$
\begin{aligned}
& I_{1}^{3} \equiv R^{3}, \quad I_{2}^{3} \equiv R R_{\mu \nu} R^{\mu \nu}, \quad I_{3}^{3} \equiv R_{\nu \alpha} R_{\mu}^{\nu} R^{\alpha \mu}, \\
& I_{4}^{3} \equiv R_{\nu \alpha} R_{\mu \beta} R^{\nu \mu \alpha \beta}, \quad I_{5}^{3} \equiv R R_{\mu \nu \alpha \beta} R^{\mu \nu \alpha \beta}, \quad I_{6}^{3} \equiv R_{\nu \alpha} R_{\beta \gamma \epsilon}{ }^{\nu} R^{\beta \gamma \epsilon \alpha}, \\
& I_{7}^{3} \equiv R_{\mu \nu \alpha \beta} R^{\mu \nu}{ }_{\gamma \epsilon} R^{\alpha \beta \gamma \epsilon}, \quad I_{8}^{3} \equiv R_{\mu \nu \alpha \beta} R^{\mu}{ }_{\gamma}{ }^{\alpha}{ }_{\epsilon} R^{\nu \gamma \beta \epsilon} .
\end{aligned}
$$

Only $I_{7}^{3}$ and $I_{8}^{3}$ contain $C^{3}$. One linear combination of these can be completed to

$$
\mathcal{L}_{3}=\frac{1}{90}\left[I_{1}^{3}-24 I_{2}^{3}+16 I_{3}^{3}+24 I_{4}^{3}+3 I_{5}^{3}-24 I_{6}^{3}+4 I_{7}^{3}-8 I_{8}^{3}\right] .
$$

The other combination of $I_{7}^{3}$ and $I_{8}^{3}$,

$$
\begin{aligned}
\mathcal{O}_{3}= & 4 I_{1}^{3}-12(n+1) I_{2}^{3}+8(3 n-1) I_{3}^{3} \\
& +12\left(n^{2}-n+2\right) I_{4}^{3}+6(n-1) I_{5}^{3}-12\left(n^{2}-1\right) I_{6}^{3} \\
& -4\left(n^{3}-3 n^{2}+2 n+2\right) I_{7}^{3}+\left(3 n^{2}-3 n-2\right) I_{8}^{3}
\end{aligned}
$$

can be shown to vanish for our geometries as required by the uniqueness arguments of the previous section, see (4.9). The remaining seven independent invariants have the explicit form:

$$
\begin{aligned}
\operatorname{tr} R^{3} & =F^{3}+G^{3}+n H^{3} \\
(\operatorname{tr} R)\left(\operatorname{tr} R^{2}\right) & =(F+G+n H)\left(F^{2}+G^{2}+n H^{2}\right), \\
(\operatorname{tr} R)^{3} & =(F+G+n H)^{3} ; \\
C^{\mu \nu}{ }_{\alpha \beta} R_{\mu}{ }^{\alpha} R_{\nu}{ }^{\beta} & =-2 \chi \frac{n-1}{n+1}(F-H)(G-H) ; \\
\left(C^{2}\right)^{\mu \nu}{ }_{\alpha \nu} R_{\mu}{ }^{\alpha} & =2 \chi^{2} \frac{n-1}{n(n+1)}[(F+G)(n-1)+2 H], \\
\left(\operatorname{tr} C^{2}\right)(\operatorname{tr} R) & =4 \chi^{2} \frac{n-1}{n+1}(F+G+n H) ; \\
\left(\operatorname{tr} C^{3}\right) & =\left(-2 \chi \frac{n-1}{n+1}\right)^{3}\left[1-\frac{2}{n^{2}}+\frac{4}{n^{2}(n-1)^{2}}\right] .
\end{aligned}
$$


At order $k=4$, there are in general 26 invariants [5], which we do not list here. Of these, seven contain $C^{4}$, the single surviving combination of which can be completed $[11,12]$ to make

$$
\begin{aligned}
\mathcal{L}_{4}=96 & {\left[\frac{1}{2}\left(R^{\mu \nu}{ }_{\alpha \beta} R^{\alpha \beta}{ }_{\mu \nu}\right)^{2}+R^{\mu \nu}{ }_{\alpha \beta} R^{\alpha \beta}{ }_{\epsilon \gamma} R^{\epsilon \gamma}{ }_{\kappa \zeta} R^{\kappa \zeta}{ }_{\mu \nu}+8 R^{\mu \nu}{ }_{\alpha \beta} R^{\beta \gamma}{ }_{\nu \epsilon} R^{\epsilon \kappa}{ }_{\gamma \zeta} R^{\zeta \alpha}{ }_{\kappa \mu}\right.} \\
& -8 R^{\mu \nu}{ }_{\epsilon \gamma} R^{\gamma \kappa}{ }_{\mu \nu} R^{\alpha \beta}{ }_{\kappa \zeta} R^{\zeta \epsilon}{ }_{\alpha \beta}+16 R^{\epsilon \kappa}{ }_{\mu \nu} R^{\mu \nu}{ }_{\alpha \beta} R^{\alpha \zeta}{ }_{\epsilon \gamma} R^{\beta \gamma}{ }_{\kappa \zeta} \\
& \left.+16 R^{\alpha \kappa}{ }_{\mu \nu} R^{\mu \epsilon}{ }_{\alpha \beta} R^{\nu \zeta}{ }_{\epsilon \gamma} R^{\beta \gamma}{ }_{\kappa \zeta}+\left(\text { terms involving } R \text { and } R_{\mu}{ }^{\nu}\right)\right] .
\end{aligned}
$$

Of the remaining 19 terms, six more also vanish. Finally, we are left with 14 linearly independent invariants, including all possible non-Weyl terms:

$$
\begin{aligned}
& \operatorname{tr} R^{4}, \quad\left(\operatorname{tr} R^{3}\right)(\operatorname{tr} R), \quad\left(\operatorname{tr} R^{2}\right)^{2}, \quad\left(\operatorname{tr} R^{2}\right)(\operatorname{tr} R)^{2}, \quad(\operatorname{tr} R)^{4} ; \\
& C^{\mu \nu}{ }_{\alpha \beta}\left(R^{2}\right)_{\mu}{ }^{\alpha} R_{\nu}{ }^{\beta}, \quad C^{\mu \nu}{ }_{\alpha \beta} R_{\mu}{ }^{\alpha} R_{\nu}{ }^{\beta}(\operatorname{tr} R) ; \\
& \left(\operatorname{tr} C^{2}\right)\left(\operatorname{tr} R^{2}\right), \quad\left(\operatorname{tr} C^{2}\right)(\operatorname{tr} R)^{2}, \quad\left(C^{2}\right)^{\mu \nu}{ }_{\alpha \nu}\left(R^{2}\right)_{\mu}{ }^{\alpha}, \quad\left(C^{2}\right)^{\mu \nu}{ }_{\alpha \beta} R_{\mu}{ }^{\alpha} R_{\nu}{ }^{\beta} ; \\
& \left(C^{3}\right)^{\mu \nu}{ }_{\alpha \nu} R_{\mu}{ }^{\alpha}, \quad\left(\operatorname{tr} C^{3}\right)(\operatorname{tr} R) ; \\
& \operatorname{tr} C^{4} .
\end{aligned}
$$

This is the general pattern. Maximal reduction occurs for Weyl ${ }^{k}$; any possible contraction of indices in the $k$ occurrences of the Weyl tensor is proportional to a single invariant

$$
\left(\operatorname{tr} C^{k}\right) \propto \chi^{k}
$$

With one Ricci, there are two independent invariants

$$
\left(C^{k}\right)^{\mu \nu}{ }_{\alpha \nu} R_{\mu}^{\alpha}, \quad\left(\operatorname{tr} C^{k}\right)(\operatorname{tr} R)
$$

there are no others since the only combinations linear in $F, G, H$ and symmetric under $F \leftrightarrow G$ are $(F+G)$ and $H$. Similarly, there can be at most four invariants with two Riccis, since the $F \leftrightarrow G$ symmetric bilinears in $F, G, H$ are $F^{2}+G^{2}, F G,(F+G) H$, and $H^{2}$; for example one can take

$$
\left(\operatorname{tr} C^{k-2}\right)\left(\operatorname{tr} R^{2}\right), \quad\left(\operatorname{tr} C^{k-2}\right)(\operatorname{tr} R)^{2}, \quad\left(C^{k-2}\right)^{\mu \nu}{ }_{\alpha \nu}\left(R^{2}\right)_{\mu}{ }^{\alpha}, \quad\left(C^{k-2}\right)^{\mu \nu}{ }_{\alpha \beta} R_{\mu}{ }^{\alpha} R_{\nu}{ }^{\beta} .
$$

For low $D$ we may get fewer linearly independent invariants. Thus, in the case of $D=4$ the identity $\left(C^{2}\right)_{\nu \alpha}^{\mu \alpha}=\frac{1}{4} \delta_{\nu}^{\mu}\left(C^{2}\right)^{\alpha \beta}{ }_{\alpha \beta}$ relates the two scalars in (5.13), and there are similar relations in (5.14). Indeed, $D=4$ is both special, and of course the most extensively studied [13]. The greatest variety occurs for scalars made entirely out of Ricci, which are of the form

$$
\left(\operatorname{tr} R^{k_{1}}\right)\left(\operatorname{tr} R^{k_{2}}\right)\left(\operatorname{tr} R^{k_{3}}\right) \ldots, \quad k_{1}+k_{2}+k_{3}+\ldots=k
$$


Here, $k_{i} \leq D$ since the characteristic polynomial provides a relation, $0=c_{R}(R)=$ $R^{D}+c_{1} R^{D-1}+c_{2} R^{D-2}+\ldots+c_{D-1} R+c_{D}$ which expresses $R^{D}$ in terms of lower powers of $R$. Moreover, since $n=D-2$ out of $D$ eigenvalues of $R$ are degenerate, vanishing discriminants provide additional relations. For example, the discriminant of $c_{R}(R)$, namely $\mathcal{D} \equiv \prod_{i<j}\left(\lambda_{i}-\lambda_{j}\right)^{2}=\mathcal{D}\left(c_{1}, \ldots, c_{D}\right)$ where $\lambda_{i}$ are the eigenvalues of $R$, is a homogeneous polynomial in the entries of $R$, of degree $D(D-1)$. When some of the $\lambda_{i}$ 's coincide, $\mathcal{D}\left(c_{1}, \ldots, c_{D}\right)=0$ and we have an additional relation on the order $k=D(D-1)$ invariants. In fact, since $n=D-2$ eigenvalues coincide, there are $n(n-1)$ such relations for $k=D(D-1), D(D-1)-2, D(D-1)-4$, etc.

We see that things get more complicated with growing powers of Ricci. A crude upper bound on the number of possible invariants made with $l$ Riccis $(3.5)$ and $(k-l)$ Weyls (3.6), is the number of linearly independent homogeneous polynomials of order $l$ in the three variables $F, G$, and $H$ which are symmetric under $F \leftrightarrow G$.

\section{$6 \quad$ Less symmetry?}

So far we have relied heavily on maximal symmetry. In this section we show why this is so special by considering the next simplest case of static axially symmetric $D=n+4$ dimensional metrics,

$$
\begin{aligned}
d s^{2} & =-e^{2 U}(d t+\mathcal{A} d \phi)^{2}+e^{-2 U} r^{2} d \phi^{2}+e^{-2 V}\left(d r^{2}+d z^{2}\right)+e^{-2 Y} d \Omega_{n}^{2} \\
& =-\omega_{\hat{t}}^{2}+\omega_{\hat{\phi}}^{2}+\omega_{\hat{r}}^{2}+\omega_{\hat{z}}^{2}+\sum_{i=1}^{n} \omega_{\hat{i}}^{2}
\end{aligned}
$$

the four functions $\mathcal{A}, U, V$, and $Y$ depend on both $r$ and $z$. The problem now becomes much less tractable. The connection one-forms (2.3) are

$$
\begin{aligned}
& \omega_{\hat{t} \hat{r}}=e^{V} U_{, r} \omega_{\hat{t}}+\frac{1}{2 r} e^{V+2 U} \mathcal{A}_{, r} \omega_{\hat{\phi}}, \omega_{\hat{t} \hat{z}}=e^{V} U_{, z} \omega_{\hat{t}}+\frac{1}{2 r} e^{V+2 U} \mathcal{A}_{, z} \omega_{\hat{\phi}}, \\
& \omega_{\hat{t} \hat{\phi}}=-\frac{1}{2 r} e^{V+2 U}\left(\mathcal{A}_{r r} \omega_{\hat{r}}+\mathcal{A}_{, z} \omega_{\hat{z}}\right), \omega_{\hat{r} \hat{z}}=e^{V} V_{, z} \omega_{\hat{r}}+e^{V} V_{, r} \omega_{\hat{z}}, \\
& \omega_{\hat{r} \hat{\phi}}=\frac{1}{2 r} e^{V+2 U} \mathcal{A}_{, r} \omega_{\hat{t}}+e^{V}\left(U_{, r}-\frac{1}{r}\right) \omega_{\hat{\phi}}, \omega_{\hat{z} \hat{\phi}}=\frac{1}{2 r} e^{V+2 U} \mathcal{A}_{, z} \omega_{\hat{t}}+e^{V} U_{, z} \omega_{\hat{\phi}}, \\
& \omega_{\hat{r} \hat{i}}=e^{V} Y_{, r} \omega_{\hat{i}}, \omega_{\hat{z} \hat{i}}=e^{V} Y_{, z} \omega_{\hat{i}}, \omega_{\hat{t} \hat{i}}=\omega_{\hat{\phi} \hat{i}}=0 .
\end{aligned}
$$


It has the following block diagonal form:

$$
R_{\hat{\mu} \hat{\nu}}=\left(\begin{array}{lllll}
R_{\hat{t} \hat{t}} & R_{\hat{t} \hat{\phi}} & & & \\
R_{\hat{\phi} \hat{t}} & R_{\hat{\phi} \hat{\phi}} & & & \\
& & R_{\hat{r} \hat{r}} & R_{\hat{r} \hat{z}} & \\
& & R_{\hat{z} \hat{r}} & R_{\hat{z} \hat{z}} & \\
& & & & \psi_{n} \mathbf{1}_{n \times n}
\end{array}\right)
$$

with all other entries zero. The Ricci tensor has 7 independent components, at least for $D \geq 5$ :

$$
\begin{aligned}
& R_{\hat{t} \hat{t}}=e^{2 V}\left[\frac{e^{4 U}}{2 r^{2}}\left(\mathcal{A}_{, r}^{2}+\mathcal{A}_{, z}^{2}\right)+\frac{1}{r} U_{, r}+\left(U_{, r r}+U_{, z z}\right)-n\left(U_{, r} Y_{, r}+U_{, z} Y_{, z}\right)\right], \\
& R_{\hat{\phi} \hat{\phi}}=e^{2 V}\left[\frac{e^{4 U}}{2 r^{2}}\left(\mathcal{A}_{, r}^{2}+\mathcal{A}_{, z}^{2}\right)+\frac{1}{r} U_{, r}+\left(U_{, r r}+U_{, z z}\right)-n\left(U_{, r} Y_{, r}+U_{, z} Y_{, z}-\frac{1}{r} Y_{, r}\right)\right], \\
& R_{\hat{t} \hat{\phi}}=R_{\hat{\phi} \hat{t}}=\frac{e^{2(U+V)}}{2 r}\left[\left(\mathcal{A}_{, r r}+\mathcal{A}_{, z z}\right)-\frac{1}{r} \mathcal{A}_{, r}+4\left(U_{, r} \mathcal{A}_{, r}+U_{, z} \mathcal{A}_{, z}\right)-n\left(Y_{, r} \mathcal{A}_{, r}+Y_{, z} \mathcal{A}_{, z}\right)\right] ; \\
& R_{\hat{r} \hat{r}}=e^{2 V}\left[\frac{e^{4 U}}{2 r^{2}} \mathcal{A}_{, r}^{2}+\left(V_{, r r}+V_{, z z}\right)-2 U_{, r}^{2}+\frac{1}{r}\left(2 U_{, r}-V_{, r}\right)\right. \\
& \left.+n\left(Y_{, r r}-Y_{, r}^{2}+V_{, r} Y_{, r}-V_{, z} Y_{, z}\right)\right], \\
& R_{\hat{z} \hat{z}}=e^{2 V}\left[\frac{e^{4 U}}{2 r^{2}} \mathcal{A}_{, z}^{2}+\left(V_{, r r}+V_{, z z}\right)-2 U_{, z}^{2}+\frac{1}{r} V_{, r}\right. \\
& \left.+n\left(Y_{, z z}-Y_{, z}^{2}+V_{, z} Y_{, z}-V_{, r} Y_{, r}\right)\right], \\
& R_{\hat{r} \hat{z}}=R_{\hat{z} \hat{r}}=e^{2 V}\left[\frac{e^{4 U}}{2 r^{2}} \mathcal{A}_{, r} \mathcal{A}_{, z}+\frac{1}{r}\left(U_{, z}-V_{, z}\right)-2 U_{, r} U_{, z}\right. \\
& \left.+n\left(V_{, r} Y_{, z}+V_{, z} Y_{, r}-Y_{, r} Y_{, z}+Y_{, r z}\right)\right] ; \\
& \psi_{n}=(n-1) e^{2 Y}+e^{2 V}\left[\frac{1}{r} Y_{, r}+\left(Y_{, r r}+Y_{, z z}\right)-n\left(Y_{, r}^{2}+Y_{, z}^{2}\right)\right] \text {. }
\end{aligned}
$$

More importantly, the Weyl tensor no longer depends on a single function, so one can construct more than one linearly independent combination of Weyl ${ }^{k}$ up to terms involving Riccis, and there is no longer any decomposition of the form (4.9). So indeed spherical symmetry is very crucial for our simplifications.

\section{$7 \quad$ Effective actions}

For the purpose of effective actions, there are basically three types of terms at any order $k$. The first are the pure Lovelock invariants $\mathcal{L}_{k}$, with actions (4.5); their solutions [11] are direct extensions of the single Gauss-Bonnet metric [10]. At the other extreme, all actions with two or more Ricci tensors permit Ricci-flat, i.e. Schwarzschild solutions, 
since the Euler-Lagrange equations always contain at least one Ricci tensor or scalar. Indeed, they may even allow Schwarzschild-deSitter metrics where $R_{\mu \nu}=\lambda g_{\mu \nu}$ provided the relative coefficients $a_{k}$ in (4.5) are appropriately chosen. Being of higher derivative order, they will also have other, non-Einstein space, solutions. The third, intermediate, class consists of actions of the form $\int\left(d^{D} x\right) \sqrt{-g} R_{\mu \nu} f^{\mu \nu}$, with $f^{\mu \nu} \equiv \alpha\left(C^{k-1}\right)^{\mu \nu}+\beta g^{\mu \nu} \operatorname{tr} C^{k-1}$. These actions do not have Ricci-flat solutions: $R_{\mu \nu}=0$ implies Schwarzschild, completely determining $C_{\alpha \beta}^{\mu \nu}$ as well; the resulting $f^{\mu \nu}$ is then incompatible with the (Ricci-flat) equations of motion

$$
\nabla_{\alpha} \nabla^{\mu} f^{\alpha \nu}+\nabla_{\alpha} \nabla^{\mu} f^{\nu \alpha}-\nabla_{\alpha} \nabla^{\alpha} f^{\mu \nu}-g^{\mu \nu} \nabla_{\alpha} \nabla_{\beta} f^{\alpha \beta}=0
$$

We have not been able to find explicit solutions here, apart from the obvious $\left(C^{\mu \nu}{ }_{\alpha \beta}=0\right)$ vacua: arbitrary $(\mathrm{A}) \mathrm{dS}$ spaces.

To summarize, we have presented a framework for categorizing the local invariants of static spherically symmetric geometries. This made possible, in particular, a simple division, into three general classes, of the local actions beyond Einstein's, such as those implied by string theory expansions. These were distinguished by their Ricci tensor dependence. It would be interesting to see if any of these candidates give rise to novel, post-Schwarzschild/Lovelock effects.

\section{Acknowledgements}

We thank Marta Gomez-Reino for computer help. This work was supported in part by NSF grant PHY04-01667.

\section{Appendix A Powers of Curvature}

In this Appendix we list some invariants used in Sections 3 and 4. Powers of Ricci, Riemann and Weyl are given by

$$
\begin{aligned}
& \left(R^{k}\right)_{\alpha}{ }^{\beta} \equiv R_{\alpha}^{\alpha_{2}} R_{\alpha_{2}}^{\alpha_{3}} \ldots R_{\alpha_{k}}{ }^{\beta}=\left(F^{k} \tau+G^{k} \rho+H^{k} \sigma\right)_{\alpha}^{\beta}, \\
& \left(R^{k}\right)^{\alpha \beta}{ }_{\mu \nu} \equiv R_{\epsilon_{2} \gamma_{2}}^{\alpha \beta} R_{\epsilon_{3} \gamma_{3}}^{\epsilon_{2} \gamma_{2}} R_{\mu \nu}^{\epsilon_{k} \gamma_{k}} \\
& =2^{k}\left[(-A)^{k} \cdot 2 \tau_{[\mu}^{[\alpha} \rho_{\nu]}^{\beta]}+B^{k} \cdot 2 \tau_{[\mu}^{[\alpha} \sigma_{\nu]}^{\beta]}+C^{k} \cdot 2 \rho_{[\mu}^{[\alpha} \sigma_{\nu]}^{\beta]}+\psi^{k} \cdot \sigma_{[\mu}^{[\alpha} \sigma_{\nu]}^{\beta]}\right] \text {, } \\
& \left(C^{k}\right)^{\alpha \beta}{ }_{\mu \nu} \equiv C_{\epsilon_{2} \gamma_{2}}^{\alpha \beta} C_{\epsilon_{3} \gamma_{3}}^{\epsilon_{2} \gamma_{2}} C^{\epsilon_{k} \gamma_{k}}{ }_{\mu \nu} \\
& =\left(-2 \chi \frac{n-1}{n+1}\right)^{k}\left[2 \tau_{[\mu}^{[\alpha} \rho_{\nu]}^{\beta]}+2\left(-\frac{1}{n}\right)^{k}\left(\tau_{[\mu}^{[\alpha} \sigma_{\nu]}^{\beta]}+\rho_{[\mu}^{[\alpha} \sigma_{\nu]}^{\beta]}\right)+\left(\frac{2}{n(n-1)}\right)^{k} \sigma_{[\mu}^{[\alpha} \sigma_{\nu]}^{\beta]}\right] \text {. }
\end{aligned}
$$


Here, $k=0$ is the identity tensor of the appropriate symmetry type, e.g. $\left(R^{0}\right)_{\alpha}{ }^{\beta}=\delta_{\alpha}{ }^{\beta}$ and $\left(R^{0}\right)^{\alpha \beta}{ }_{\mu \nu}=2 \tau_{[\mu}^{[\alpha} \rho_{\nu]}^{\beta]}+2 \tau_{[\mu}^{[\alpha} \sigma_{\nu]}^{\beta]}+2 \rho_{[\mu}^{[\alpha} \sigma_{\nu]}^{\beta]}+\sigma_{[\mu}^{[\alpha} \sigma_{\nu]}^{\beta]}$.

Contraction of these produces

$$
\begin{aligned}
\left(R^{k}\right)_{\alpha}^{\alpha} & =F^{k}+G^{k}+H^{k} n, \\
\left(R^{k}\right)^{\alpha \beta}{ }_{\gamma \beta} & =2^{k-1}\left[\tau\left((-A)^{k}+n B^{k}\right)+\rho\left((-A)^{k}+n C^{k}\right)+\sigma\left(B^{k}+C^{k}+(n-1) \psi^{k}\right)\right]_{\gamma}^{\alpha} \\
\left(C^{k}\right)_{\gamma \beta}^{\alpha \beta} & =\frac{1}{2}\left(-2 \chi \frac{n-1}{n+1}\right)^{k}\left[(\tau+\rho)\left(1-(-n)^{1-k}\right)+\frac{2 \sigma}{n^{k}}\left(\left(\frac{2}{n-1}\right)^{k-1}-(-1)^{k-1}\right)\right]_{\gamma}^{\alpha}
\end{aligned}
$$

and in particular at $D=4$, we recover the identity

$$
\left.\left(C^{2}\right)^{\alpha \beta}{ }_{\gamma \beta}\right|_{n=2}=\left(-2 \chi \frac{1}{3}\right)^{2} \frac{3}{4}[\tau+\rho+\sigma]_{\gamma}^{\alpha}=\frac{3}{4} \delta_{\gamma}^{\alpha}\left(-2 \chi \frac{1}{3}\right)^{2}=\frac{1}{4} \delta_{\gamma}^{\alpha}\left(C^{2}\right)^{\mu \nu}{ }_{\mu \nu} .
$$

Some examples of resulting invariants are

$$
\begin{aligned}
\left(C^{k}\right)^{\alpha \beta}{ }_{\alpha \beta} & =\left(-2 \chi \frac{n-1}{n+1}\right)^{k}\left[1-\frac{2(-1)^{k-1}}{n^{k-1}}+\frac{2^{k-1}}{n^{k-1}(n-1)^{k-1}}\right], \\
\left(C^{k}\right)^{\alpha \beta}{ }_{\alpha \beta} R_{\mu}^{\mu} & =\left(-2 \chi \frac{n-1}{n+1}\right)^{k}\left[1-\frac{2(-1)^{k-1}}{n^{k-1}}+\frac{2^{k-1}}{n^{k-1}(n-1)^{k-1}}\right](F+G+n H),(\mathrm{A} .9) \\
\left(C^{k}\right)^{\alpha \beta}{ }_{\gamma \beta} R_{\alpha}^{\gamma} & =\left(-2 \chi \frac{n-1}{n+1}\right)^{k}\left[\frac{F+G}{2}\left(1-\frac{(-1)^{k-1}}{n^{k-1}}\right)+\frac{H}{n^{k-1}}\left(\frac{2^{k-1}}{(n-1)^{k-1}}-(-1)^{k-1}\right)\right] .
\end{aligned}
$$

To get the equivalents of (A.9) and (A.10) for $R^{k}$ instead of $R$, all we have to do is replace $F, G$, and $H$ in their right hand sides by $F^{k}, G^{k}$, and $H^{k}$ respectively.

\section{References}

[1] S. Deser, J. H. Kay and K. S. Stelle, "Renormalizability Properties Of Supergravity," Phys. Rev. Lett. 38, 527 (1977).

[2] Z. Bern, L. J. Dixon, D. C. Dunbar, M. Perelstein and J. S. Rozowsky, "On the relationship between Yang-Mills theory and gravity and its implication for ultraviolet divergences," Nucl. Phys. B 530, 401 (1998) [arXiv:hep-th/9802162].

[3] S. Deser, J. S. Franklin and D. Seminara, "Graviton graviton scattering, BelRobinson and energy (pseudo)-tensors," Class. Quant. Grav. 16, 2815 (1999) [arXiv:gr-qc/9905021]. 
[4] S. Deser and D. Seminara, "Tree amplitudes and two-loop counterterms in D $=11$ supergravity," Phys. Rev. D 62, 084010 (2000) [arXiv:hep-th/0002241].

[5] S. A. Fulling, R. C. King, B. G. Wybourne and C. J. Cummings, "Normal forms for tensor polynomials: I. The Riemann tensor," Class. Quantum Grav. 9 (1992) 1151-1197

[6] C. W. Misner, K. S. Thorne, and J. A. Wheeler, Gravitation, Chapter 14 (W. H. Freeman, San Francisco, 1973)

[7] R. S. Palais, "The principle of symmetric criticality," Comm. Math. Phys. 69, 19 (1979)

[8] S. Deser and B. Tekin, "Shortcuts to high symmetry solutions in gravitational theories," Class. Quant. Grav. 20, 4877 (2003) [arXiv:gr-qc/0306114].

[9] M. Banados, C. Teitelboim and J. Zanelli, "Dimensionally continued black holes," Phys. Rev. D 49, 975 (1994) [arXiv:gr-qc/9307033].

[10] D. G. Boulware and S. Deser, "String Generated Gravity Models," Phys. Rev. Lett. 55, 2656 (1985).

[11] R. C. Myers, "Superstring Gravity And Black Holes," Nucl. Phys. B 289, 701 (1987).

[12] James T. Wheeler, "Symmetric solutions to the Gauss-Bonnet extended Einstein equations," NPB 268 (1986) 737-746

[13] H. Stephani, D. Kramer, M. Maccallum, C. Hoenselaers, E. Herlt Exact Solutions Of Einstein's Field Equations, (Cambridge University Press, 2003) 